\title{
Characterization, dietary habits and nutritional intake of omnivorous, lacto-ovo vegetarian and vegan runners - a pilot study
}

Josefine Nebl ${ }^{1}$, Jan Philipp Schuchardt ${ }^{1}$, Paulina Wasserfurth ${ }^{1}$, Sven Haufe ${ }^{2}$, Julian Eigendorf ${ }^{2}$, Uwe Tegtbur ${ }^{2}$ and Andreas Hahn ${ }^{1 *}$ (D)

\begin{abstract}
Background: The number of people preferring plant-based nutrition is growing continuously in the western world. Vegetarianism and veganism are also becoming increasingly popular among individuals participating in sport. However, whether recreationally active vegetarian and vegan populations can meet their nutritional needs is not clear.

Methods: The purpose of this cross-sectional study was to compare the nutrient intake of omnivorous (OMN, $n=$ 27), lacto-ovo vegetarian ( $L O V, n=25)$ and vegan $(V E G, n=27)$ recreational runners (two to five training sessions per week) with intake recommendations of the German, Austrian and Swiss Nutrition Societies (Deutsche, Österreichische und Schweizerische Gesellschaften für Ernährung, D-A-CH) for the general population. Lifestyle factors and supplement intake were examined via questionnaires; dietary habits and nutrient intake were determined based on 3-day dietary records.

Results: More than half of each group did not reach the recommended energy intake (OMN: 10.4, 8.70-12.1; LOV: 9.67, 8.55-10.8; VEG: 10.2, 9.12-11.3 MJ). Carbohydrate intake was slightly below the recommendations of > 50 EN\% in OMN (46.7, 43.6-49.8 EN\%), while LOV (49.4, 45.5-53.3 EN\%) and VEG (55.2, 51.4-59.0 EN\%) consumed adequate amounts $(p=0.003)$. The recommended protein intake of $0.8 \mathrm{~g} / \mathrm{kg}$ body weight (D-A-CH) was exceeded in all three groups (OMN: 1.50, 1.27-1.66; LOV: 1.34, 1.09-1.56; VEG: 1.25; 1.07-1.42 g/kg BW; $p=0.047)$. Only VEG (26.3, 22.7-29.8 EN\%) did not achieve the recommended fat intake of $30 \mathrm{EN} \%$. The supply of micronutrients, such as vitamin D and cobalamin, was dependent on supplement intake. Additionally, female OMN and LOV achieved the recommended daily intake of $15 \mathrm{mg}$ iron only after supplementation, while VEG consumed adequate amounts solely via food.

Conclusion: All three groups were sufficiently supplied with most nutrients despite the exceptions mentioned above. The VEG group even showed advantages in nutrient intake (e.g. carbohydrates, fiber and iron) in comparison to the other groups. However, the demand for energy and several macro- and micronutrients might be higher for athletes. Thus, it is also necessary to analyze the endogenous status of nutrients to evaluate the influence of a vegetarian and vegan diet on the nutrient supply of athletes.
\end{abstract}

Trial registration: German Clinical Trial Register (DRKS00012377), registered on April 28, 2017.

Keywords: Recreational endurance athletes, Plant-based diets; nutrient supply, Vegetarianism, Veganism, Nutrient survey

\footnotetext{
* Correspondence: hahn@nutrition.uni-hannover.de

${ }^{1}$ Faculty of Natural Sciences, Institute of Food Science and Human Nutrition,

Leibniz University Hannover, 30167 Hannover, Germany

Full list of author information is available at the end of the article
}

(c) The Author(s). 2019 Open Access This article is distributed under the terms of the Creative Commons Attribution 4.0 International License (http://creativecommons.org/licenses/by/4.0/), which permits unrestricted use, distribution, and reproduction in any medium, provided you give appropriate credit to the original author(s) and the source, provide a link to the Creative Commons license, and indicate if changes were made. The Creative Commons Public Domain Dedication waiver (http://creativecommons.org/publicdomain/zero/1.0/) applies to the data made available in this article, unless otherwise stated. 


\section{Background}

Plant-based diets, especially vegetarianism and veganism, are increasingly gaining popularity in the western world. These alternative diets are characterized by a predominance of foodstuffs derived from plants in varying amounts and range from abstaining meat, meat products and fish to complete rejection of animal products such as vegans (VEG) $[1,2]$. About 4.3 to $10 \%$ of the population in Germany are estimated to be vegetarians, whereas the number of VEG is estimated at $1.6 \%$ [3-5]. Switzerland, Italy, Austria and the UK have a similar number of vegetarians as Germany at 9-11\% [6]. In the United States, only $5 \%$ of the population is considered vegetarian [7], however, this is still more than 16 million people.

It is undisputed that a lacto-ovo vegetarian (LOV) diet based on a broad variety of foods generally ensures the supply of (nearly) all nutrients in adults $[1,8,9]$ and has favorable effects on the cardiometabolic risk compared to the usual mixed diet [10-14]. Moreover, plant-based diets show beneficial associations with obesity, type 2 diabetes, hypertension and cancer [15-18], although healthy omnivore $(\mathrm{OMN})$ diets can achieve similar effects [19]. Consequently, several nutrition societies recommend LOV diets as a healthy diet for all stages of life $[8,20-22]$. By contrast, strict VEG nutrition is viewed as critical due to the risk for an undersupply with critical nutrients such as protein, long-chain n3 fatty acids, riboflavin, cobalamin, vitamin D, calcium, iron and zinc [23]. Thorough planning and engagement with a VEG diet are required to adjust the nutrient supply and meet the needs in different population groups.

A balanced diet also plays an important role for athletes. The impact of a plant-based diet on the health and performance of athletes is becoming a growing interest [4]. However, data on the prevalence of vegetarians or VEG as recreational and professional athletes are still sparse and only a few studies have investigated the nutritional status of vegetarian athletes $[24,25,26]$. Therefore, it is of great importance to investigate the nutritional status of athletes using data on dietary habits combined with analytical data on the nutrient status and functional outcomes. Such findings enable an evaluation of whether athletes who follow plant-based diets can meet their nutritional needs or show nutrient imbalances. Furthermore, such data form the basis for assessing the relationship of a plant-based diet with the body composition, the antioxidant and immunological capacity and, ultimately, with the health and performance of athletes [24, 26, 27]. Present studies investigating the relationship between a vegetarian and VEG diet and exercise do not differentiate between vegetarians and VEG [26], are outdated [28], questionnaire-based [25, 29, 30] or do not contain nutritional assessment including biochemical markers [31, 32].
The nutrient supply status of athletes consuming a balanced mixed diet including animal-based foods can usually be classified as safe, including critical nutrients. However, there is lack of scientific data investigating the question of whether vegetarian and especially VEG athletes are undersupplied with critical nutrients, and whether this affects health and performance. To date, no data exist on the nutritional and athletic conditions of VEG recreational runners and there are no recommendations regarding nutrient intake for LOV and VEG athletes. Therefore, in order to fill the knowledge gap between nutrient intake, status and performance, the novel approach of this study is to compare the dietary habits, nutritional intake, body composition and performance diagnostics of VEG and LOV recreational runners with OMN runners. We present here a comparison of the nutritional supply status of these three groups and a comparison with reference values of the German, Austrian and Swiss Nutrition Societies for healthy adults (Deutsche, Österreichische und Schweizerische Gesellschaft für Ernährung: D-A-CH) [33]. These data may serve as a first basis to determine specific recommendations regarding the nutrient intake for vegetarian and vegan athletes in the future.

\section{Methods \\ Participants}

This cross-sectional study was conducted at the Institute of Food Science and Human Nutrition, Leibniz University Hannover, Germany. Ethical approval was provided by the Ethics Committee at the Medical Chamber of Lower Saxony (Hannover, Germany). The study was conducted in accordance with the Declaration of Helsinki. All subjects gave their written informed consent. The study was registered in the German Clinical Trial Register (DRKS00012377).

Eighty-one healthy recreational runners (mean age: $27.5 \pm 4.14$ yr., height: $1.75 \pm 0.80 \mathrm{~m}$, body mass: $67.7 \pm$ $9.56 \mathrm{~kg}$, BMI: $22.0 \pm 1.94 \mathrm{~kg} / \mathrm{m}^{2}, \mathrm{~m}=31, \mathrm{f}=50$ ) aged between 18 and 35 years were recruited from the general population in Hannover, Germany, via local running events, online running communities and online vegetarian and VEG communities.

The eligibility of subjects was assessed using questionnaires. Participants were selected based on the following inclusion criteria: OMN, LOV or VEG diet for at least half a year, body mass index (BMI) between 18.5 and $25.0 \mathrm{~kg} /$ $\mathrm{m}^{2}$ and run regularly two to five times per week for at least 30-60 min. Regular running sessions were documented via self-reporting data. The following criteria led to exclusion: Any cardiovascular, metabolic or malignant disease, diseases of the gastrointestinal tract, pregnancy, food intolerances and addiction to drugs or alcohol. Participants were allowed to take dietary supplements, but 
the use of performance-enhancing substances (e.g. alkaline salts, creatine) led to exclusion.

\section{Methods and examination procedure}

A questionnaire, which included food groups the participants usually consume, had to be completed to categorize subjects as OMN, LOV and VEG recreational athletes.

Participants were matched according to age and gender. Subjects who were included in the study collective were invited to an examination. Prior to the examination, subjects fulfilled a 3-day dietary record over three consecutive days, including 2 week days and one weekend day. The nutritional diaries were checked by nutritionists for completeness, readability and plausibility. Ambiguities were clarified with subjects if necessary. Seventy-nine out of 81 participants returned the completed dietary record. The following food groups were analyzed: Meat, meat products and sausages, fish and seafood, milk and dairy products, eggs, fat and oil, whole grain products, cereal products, pastries, potatoes, vegetables, legumes, soy, fresh fruits, nuts and seeds, sweets, alcoholic drinks, alcohol, nonalcoholic beverages, coffee, tea and fast food.

Nutrient intake was depicted in comparison to the reference values of the German, Austrian and Swiss Nutrition Societies for healthy adults (D-A-CH) [33]. Amino acid intake was compared to the reference values of the World Health Organization (WHO) [34].

All 81 participants completed a questionnaire regarding their supplement intake, status of health and running activity. Training frequency and duration were selfreported by the subjects. The determination of anthropometric data followed. The measurements of body weight $(\mathrm{BW})$ and height were carried out without shoes, respectively. Waist circumference was determined using a tape measure. The BMI was calculated using the standard formula:

$$
B M I=\frac{\text { body mass }[\mathrm{kg}]}{(\text { height }[\mathrm{m}])^{2}}
$$

\section{Data analysis and statistical methods}

The nutrition organization software PRODI6.4 (NutriScience GmbH, Freiburg, Germany) was used to analyze dietary habits, energy and nutrient intake from the 3-day dietary record. The composition of foods, which were not available in PRODI6.4 $4^{\circ}$, have been requested from the manufacturer and the results were integrated into the software. The intake of animal- and plant-based iron was also calculated with the software. The compositions of all supplementary products mentioned at the time of evaluation were researched and multiplied by the intake frequency (daily intake (factor *1), two times a week (factor *2/7), three times a week (factor *3/7), four times a week (factor *4/7), irregular intake (factor *12/365)) to calculate the average daily intake of the respective nutrients via supplements. Based on the intake frequencies above, the average daily intake for each mineral and vitamin was calculated for each subject individually.

Statistical analyses were performed using SPSS software (IBM SPSS Statistics 24.0; Chicago, IL, USA). Results are presented as mean \pm standard deviation (SD) or 95\% confidence interval (CI). Normal distribution was checked using the Kolmogorov-Smirnov test. If data were normally distributed, one-way analysis of variance (ANOVA) was used to evaluate differences in nutritional status and intake between the three diet groups. The Kruskal-Wallis test was performed to analyze data with non-normal distribution. If there were significant differences between the groups, the post hoc test with Bonferroni correction was conducted. The Mann-Whitney U test was used to examine differences between supplement users (SU) and non-supplement users (non-SU) within the groups. The chi-square test was used to compare the differences between the frequencies of the three groups. Associations between parametric data were computed via Pearson and nonparametric data via Spearman's rho correlation. $P$ values $\leq 0.05$ were interpreted as statistically significant.

\section{Results}

\section{Characterization of the study population}

Twenty-seven of the 81 runners followed OMN nutrition, 26 were LOV and 28 were VEG (Fig. 1). Men and women were equally distributed and there were no differences in the mean age and anthropometric data (Table 1). Only one female of the LOV had a waist circumference slightly over $80 \mathrm{~cm}$; all other participants had values in the reference range of $<80 \mathrm{~cm}$ for women and $<94 \mathrm{~cm}$ for men. All but one of the 27 participants of the OMN group had followed the diet for $>3$ years. By contrast, 4 out of 26 participants of the LOV group and 6 out of 28 of the VEG group had switched to their current diet between $0.5-1$ year.

Several subjects took dietary supplements. More precisely, 18 out of 28 participants (64.3\%) of the VEG, 10 out of $27(37.0 \%)$ of the OMN and 9 out of $26(34.6 \%)$ of the LOV group took supplements. Although considerably more subjects of the VEG group consumed supplements, there were no statistically significant differences between the groups. Magnesium, calcium, iron, cobalamin and vitamin D were commonly consumed supplements (Table 1). Magnesium and vitamin D were most commonly supplemented in the OMN group $(22.2 \%$ and not significant [n.s.], respectively), magnesium, iron and cobalamin in LOV (15.4\%; n.s.), and cobalamin in VEG $\left(53.9 \% ; p=0.005, \chi^{2}\right)$. 

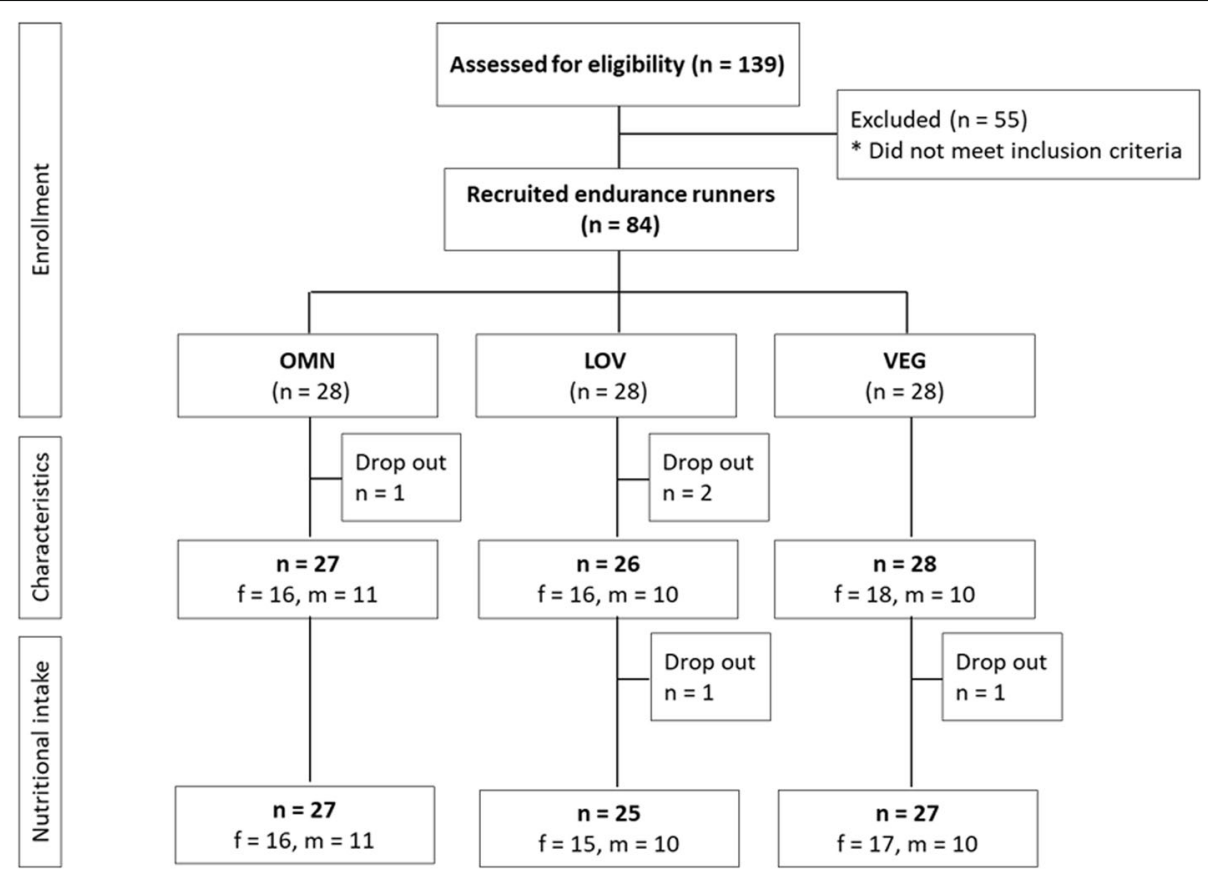

Fig. 1 Flow chart of the study population

Total nutrient intake of SU compared to non-SU was investigated (Figs. 2 and 3). Statistically significantly higher cobalamin intake in SU compared to non-SU was found in both male and female VEG $(p=0.019$ and 0.003 , respectively) as well as in female OMN $(p=0.027)$ and LOV $(p=$ $0.026)$. Magnesium $(p=0.036)$, vitamin $\mathrm{D}(p=0.018)$ and iron $(p=0.018)$ intake was statistically significantly higher in female LOV SU compared to non-SU. Male SU in OMN also showed higher iron intakes than non-SU $(p=0.004)$. The analysis of fortified food products revealed only one subject who consumed a small amount $(15 \mathrm{mg})$ of calciumenriched soy drink, which can be neglected.

None of the subjects regularly consumed tobacco. The participants showed no differences in training frequency or duration (Table 1).

\section{Dietary habits}

According to their diet, LOV and VEG consumed neither meat, meat products, fish nor seafood (Table 2). The VEG additionally waived milk, dairy products and eggs. The three groups consumed similar amounts of fat and oil, whole grain and cereal products as well as pastries. Moreover, there were no significant differences in the dietary intake of sweets, alcoholic drinks, coffee and tea. The VEG consumed significantly higher amounts of potatoes, vegetables and fresh fruit compared to LOV ( $\mathrm{p}_{\mathrm{LOV}-\mathrm{VEG}}=0.013,0.031$ and 0.041 , respectively) and OMN (pOMN-VEG $=0.017,0.000$ and 0.015 , respectively). Legumes were consumed mainly in the VEG group $(p<0.001)$, while OMN consumed the highest amounts of fast food $(p=0.016)$ (Table 2).

\section{Nutritional intake}

None of the three groups differed in terms of energy consumption (Table 3); men (OMN: 12.3, 8.36-16.1; LOV: 10.3, 8.96-11.7; VEG: 11.5, 8.97-1 3.9 M); n.s.) had a higher energy intake than women (OMN: 9.11, 7.96-10.3; LOV: 9.22, 7.51-10.9; VEG: 9.47, 8.47-10.4 MJ; n.s.), which was statistically significant for OMN $(p=0.023)$. In comparison to the recommended values for people who perform sport several times a week (age group 19-25 and 25-51, physical activity level was estimated at 1.7 [33];), only the average of female VEG and male OMN reached the recommendations. Low levels of energy intake were evident in $59.3 \%$ of OMN, $52.0 \%$ of LOV and $51.9 \%$ of VEG, with no differences in frequency distribution. No significant associations were found between energy intake and age, BMI and frequency of training.

Regarding the macronutrient intake, there were significant differences between OMN and VEG. The VEG consumed a higher percentage of carbohydrates (55.2, 51.4-59.0 energy percent, EN\%) compared to OMN (46.7, 43.6-49.8 EN\%; POMN-VEG $=0.002)$ (Table 3). Most subjects of the OMN group (70.4\%) and $50.2 \%$ of the LOV group had low levels (<50 EN\%) of carbohydrates. By contrast, most subjects (66.7\%) of the VEG group had higher levels of carbohydrates (> $55 \mathrm{EN} \%$ ). These differences were statistically significant $\left(p=0.035, \chi^{2}\right)$. The absolute intake of carbohydrates differed only slightly.

Regarding the absolute dietary protein intake, there were only minor differences between the groups 
Table 1 Characterization of the study population (mean \pm SD)

\begin{tabular}{|c|c|c|c|c|c|c|c|}
\hline & $\mathrm{OMN}(n=27)$ & $\begin{array}{l}p \text { value } \\
\text { OMN-LOV }\end{array}$ & $\operatorname{LOV}(n=26)$ & $\begin{array}{l}p \text { value } \\
\text { LOV-VEG }\end{array}$ & VEG $(n=28)$ & $\begin{array}{l}p \text { value } \\
\text { OMN-VEG }\end{array}$ & $\begin{array}{l}p \text { value } \\
3 \text { groups }\end{array}$ \\
\hline Age, years & $27.4 \pm 4.03$ & - & $27.6 \pm 4.31$ & - & $27.5 \pm 4.24$ & - & $0.968^{b}$ \\
\hline Sex & $m=11, w=16$ & - & $m=10, w=16$ & - & $m=10, w=18$ & - & $0.929^{c}$ \\
\hline $\mathrm{BMI}, \mathrm{kg} / \mathrm{m}^{2}$ & $22.3 \pm 1.74$ & - & $21.6 \pm 1.98$ & - & $22.1 \pm 2.09$ & - & $0.436^{\mathrm{b}}$ \\
\hline \multicolumn{8}{|l|}{ Waist, $\mathrm{cm}$} \\
\hline Female & $71.0 \pm 4.3$ & - & $70.1 \pm 3.8$ & - & $69.5 \pm 5.0$ & - & $0.057^{\mathrm{a}}$ \\
\hline Male & $79.5 \pm 4.3$ & - & $76.4 \pm 3.0$ & - & $80.6 \pm 4.1$ & - & $0.591^{a}$ \\
\hline $\begin{array}{l}\text { Systolic blood } \\
\text { pressure, } \mathrm{mm} \mathrm{Hg}\end{array}$ & $121 \pm 11.1$ & - & $121 \pm 13.4$ & - & $116 \pm 12.6$ & - & $0.201^{b}$ \\
\hline $\begin{array}{l}\text { Diastolic blood } \\
\text { pressure, } \mathrm{mm} \mathrm{Hg}\end{array}$ & $74.0 \pm 6.00$ & - & $72.0 \pm 4.00$ & - & $72.0 \pm 9.00$ & - & $0.457^{b}$ \\
\hline Pulse rate, bpm & $66.0 \pm 9.00$ & - & $61.0 \pm 8.00$ & - & $65.0 \pm 10.00$ & - & $0.188^{b}$ \\
\hline Duration of diet & & & & & & & $0.001^{c}$ \\
\hline$<0.5$ years, $\mathrm{n}(\%)$ & $0(0)$ & & $0(0)$ & & $0(0)$ & & \\
\hline $0.5-1$ year, n (\%) & $0(0)$ & & $4(15.4)$ & & $6(21.4)$ & & \\
\hline 1-2 years, n (\%) & $1(3.7)$ & & $3(11.5)$ & & $4(14.3)$ & & \\
\hline $2-3$ years, n (\%) & $0(0)$ & & $2(7.7)$ & & $7(25.0)$ & & \\
\hline$>3$ years, n (\%) & $26(96.3)$ & & $17(65.4)$ & & $11(39.3)$ & & \\
\hline Magnesium SU, n (\%) & $5(22.2)$ & & $4(15.4)$ & & $5(17.9)$ & & $0.710^{c}$ \\
\hline Calcium SU, n (\%) & $3(11.1)$ & & $1(3.9)$ & & $2(7.14)$ & & $0.210^{c}$ \\
\hline Iron SU, n (\%) & $3(11.1)$ & & $4(15.4)$ & & $5(17.9)$ & & $0.689^{c}$ \\
\hline Vitamin $B_{12} \mathrm{SU}, \mathrm{n}(\%)$ & $4(18.5)$ & & $4(15.4)$ & & $15(53.9)$ & & $0.005^{c}$ \\
\hline Vitamin D SU, n (\%) & $5(22.2)$ & & $1(3.9)$ & & $7(25.0)$ & & $0.078^{c}$ \\
\hline Smoker, n (\%) & $0(0)$ & & $0(0)$ & - & $0(0)$ & - & - \\
\hline $\begin{array}{l}\text { Training frequency } \\
\text { per week }\end{array}$ & $3.04 \pm 0.98$ & - & $3.24 \pm 0.88$ & - & $3.00 \pm 0.85$ & - & $0.502^{b}$ \\
\hline $\begin{array}{l}\text { Running time } \\
\text { per week (h) }\end{array}$ & $2.72 \pm 1.11$ & - & $3.38 \pm 1.43$ & - & $2.65 \pm 1.38$ & - & $0.079^{b}$ \\
\hline
\end{tabular}

OMN omnivores, LOV lacto-ovo vegetarians, VEG vegans, BMI body mass index, bpm beats per minute, SU supplement users

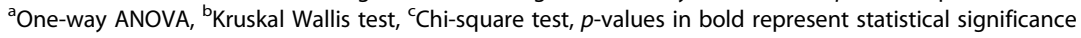

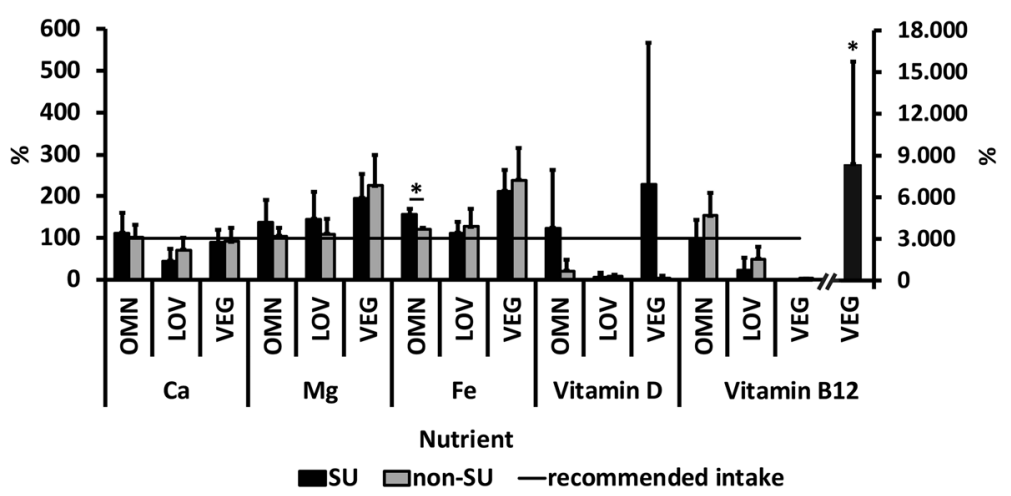

Fig. 2 Nutrient intake in relation to the reference range: Supplement users vs. non-supplement users (males; mean + SD). OMN = omnivores, LOV = lacto-ovo vegetarians, VEG = vegans, SU = supplement users, non-SU = non-supplement users, recommended intake of the German, Austrian and Swiss Nutrition Societies (Deutsche, Österreichische und Schweizerische Gesellschaften für Ernährung, D-A-CH) [33]. The error bars represent the standard deviations of the average daily nutrient intake. Differences between SU and non-SU were analyzed using the MannWhitney $U$ test. ${ }^{*} p \leq 0.05$ 


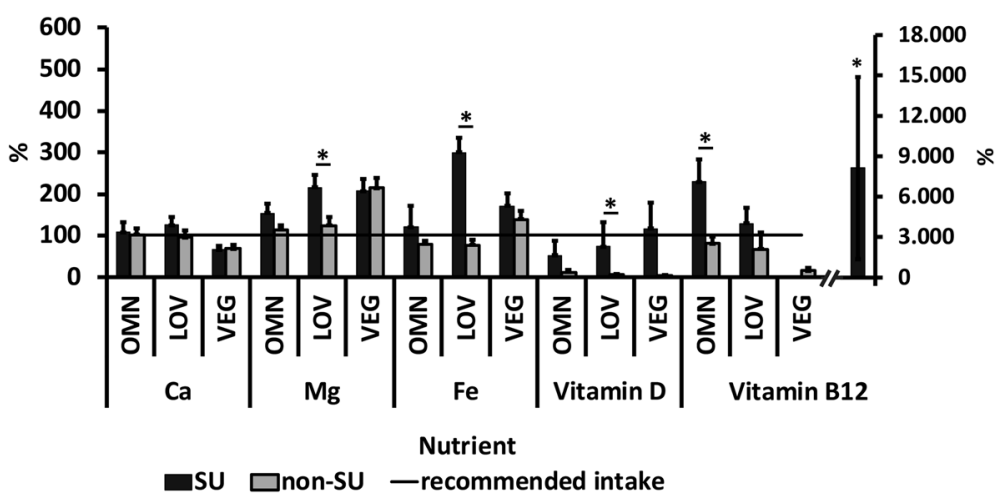

Fig. 3 Nutrient intake in relation to the reference range: SU vs. non-SU (females; mean + SD). Recommended intake of the D-A-CH [33]. The error bars represent the standard deviations of the average daily nutrient intake. Differences between SU and non-SU were analyzed using the MannWhitney $U$ test. ${ }^{*} p \leq 0.05$

(Table 3). On average, all the groups were above the reference value of $0.8 \mathrm{~g} / \mathrm{kg} \mathrm{BW}$; only one subject of the OMN group (3.70\%), two subjects of LOV $(8.00 \%)$ and two subjects of VEG (7.41\%) did not reach the recommendations (data not shown). All three groups were adequately supplied with all essential amino acids (see Additional file 1).

Considering the average relative fat intake, subjects in the OMN group ( POMN-VEG $=0.021$ ) and LOV (n.s. compared to VEG) consumed higher amounts compared to

Table 2 Mean daily intake of different food categories calculated from a 3-day dietary record

\begin{tabular}{|c|c|c|c|c|c|c|c|}
\hline$\overline{\text { Food group (g/day) }}$ & $\begin{array}{l}\mathrm{OMN} \\
(n=27)\end{array}$ & $\begin{array}{l}p \text { value } \\
\text { OMN-LOV }\end{array}$ & $\begin{array}{l}\text { LOV } \\
(n=25)\end{array}$ & $\begin{array}{l}p \text { value } \\
\text { LOV-VEG }\end{array}$ & $\begin{array}{l}\text { VEG } \\
(n=27)\end{array}$ & $\begin{array}{l}p \text { value } \\
\text { OMN-VEG }\end{array}$ & $\begin{array}{l}p \text { value } \\
3 \text { groups }\end{array}$ \\
\hline Meat & $85.8 \pm 58.8$ & $0.000^{c}$ & - & $1.000^{c}$ & - & $0.000^{c}$ & $0.000^{b}$ \\
\hline Meat products and sausages & $29.6 \pm 32.1$ & $0.000^{c}$ & - & $1.000^{c}$ & - & $0.000^{c}$ & $0.000^{\mathrm{b}}$ \\
\hline Fish and seafood & $28.7 \pm 39.9$ & $0.000^{c}$ & - & $1.000^{c}$ & - & $0.000^{c}$ & $0.000^{b}$ \\
\hline Milk and dairy products & $290 \pm 183$ & $1.000^{c}$ & $279 \pm 311$ & $0.000^{c}$ & - & $0.000^{c}$ & $0.000^{\mathrm{b}}$ \\
\hline Eggs & $23.8 \pm 37.4$ & $1.000^{c}$ & $15.8 \pm 25.0$ & $0.003^{c}$ & - & $0.000^{c}$ & $0.000^{\mathrm{b}}$ \\
\hline Fat and oil & $9.85 \pm 14.8$ & - & $10.3 \pm 12.1$ & - & $12.0 \pm 10.8$ & - & $0.228^{b}$ \\
\hline Whole grain products & $33.2 \pm 48.7$ & - & $50.6 \pm 58.8$ & - & $51.0 \pm 59.0$ & - & $0.294^{b}$ \\
\hline Cereal products & $208 \pm 141$ & - & $188 \pm 130$ & - & $220 \pm 120$ & - & $0.678^{\mathrm{a}}$ \\
\hline Pastries & $58.8 \pm 50.0$ & - & $58.0 \pm 100$ & - & $37.4 \pm 73.8$ & - & $0.067^{b}$ \\
\hline Potatoes & $44.1 \pm 79.3$ & $1.000^{c}$ & $37.5 \pm 62.3$ & $0.013^{c}$ & $118 \pm 130$ & $0.017^{c}$ & $0.005^{b}$ \\
\hline Vegetables (except potatoes, legumes) & $265 \pm 237$ & $0.511^{c}$ & $324 \pm 187$ & $0.031^{c}$ & $521 \pm 258$ & $0.000^{c}$ & $0.000^{b}$ \\
\hline Legumes (except soybeans) & $3.70 \pm 8.08$ & $0.054^{c}$ & $27.7 \pm 39.7$ & $0.092^{c}$ & $66.4 \pm 68.1$ & $0.000^{c}$ & $0.000^{\mathrm{b}}$ \\
\hline Soybeans & - & $0.007^{c}$ & $54.4 \pm 95$ & $0.031^{c}$ & $151 \pm 179$ & $0.000^{c}$ & $0.000^{\mathrm{b}}$ \\
\hline Fresh fruit & $266 \pm 160$ & $1.000^{c}$ & $288 \pm 171$ & $0.041^{c}$ & $518 \pm 404$ & $0.015^{c}$ & $0.009^{b}$ \\
\hline Nuts and seeds & $4.57 \pm 8.30$ & $0.044^{c}$ & $19.7 \pm 23.7$ & $0.578^{c}$ & $26.0 \pm 29.3$ & $0.000^{\mathrm{b}}$ & $0.001^{b}$ \\
\hline Sweets & $37.0 \pm 39.3$ & - & $38.9 \pm 44.4$ & - & $20.2 \pm 33.6$ & - & $0.148^{b}$ \\
\hline Alcoholic drinks & $131 \pm 210$ & - & $101 \pm 198$ & - & $63.0 \pm 146$ & - & $0.184^{b}$ \\
\hline Alcohol & $5.50 \pm 8.64$ & - & $3.89 \pm 6.91$ & - & $2.26 \pm 5.57$ & - & $0.345^{b}$ \\
\hline Nonalcoholic beverages (except coffee and tea) & $1103 \pm 1095$ & - & $794 \pm 1098$ & - & $1246 \pm 1258$ & - & $0.339^{b}$ \\
\hline Coffee & $170 \pm 164$ & - & $279 \pm 238$ & - & $148 \pm 198$ & - & $0.051^{b}$ \\
\hline Tea & $257 \pm 398$ & - & $181 \pm 310$ & - & $221 \pm 339$ & - & $0.999^{b}$ \\
\hline Fast food & $57.1 \pm 75.2$ & $0.063^{c}$ & $32.7 \pm 87.2$ & $1.000^{c}$ & $16.6 \pm 38.1$ & $0.025^{c}$ & $0.016^{\mathrm{b}}$ \\
\hline
\end{tabular}

All nutrients excluding dietary supplements. OMN omnivores, LOV lacto-ovo vegetarians, VEG vegans

Data are presented as mean \pm SD. ${ }^{a}$ One-way ANOVA, ${ }^{b}$ Kruskal Wallis test, ${ }^{C}$ Post hoc test, $p$-values in bold represent statistical significance 
Table 3 Absolute and relative daily energy and macronutrient intake of the study population calculated from a 3-day dietary record

\begin{tabular}{|c|c|c|c|c|c|c|c|c|}
\hline Nutrient intake & $\begin{array}{l}\text { OMN } \\
(n=27)\end{array}$ & $\begin{array}{l}p \text { value } \\
\text { OMN-LOV }\end{array}$ & $\begin{array}{l}\text { LOV } \\
(n=25)\end{array}$ & $\begin{array}{l}p \text { value } \\
\text { LOV-VEG }\end{array}$ & $\begin{array}{l}\text { VEG } \\
(n=27)\end{array}$ & $\begin{array}{l}p \text { value } \\
\text { OMN-VEG }\end{array}$ & $\begin{array}{l}p \text { value } \\
3 \text { groups }\end{array}$ & Reference values $(\mathrm{m} / \mathrm{f})^{*}$ \\
\hline \multicolumn{9}{|l|}{ Energy } \\
\hline Energy intake (MJ) & $10.4(8.70,12.1)$ & - & $9.67(8.55,10.8)$ & - & $10.2(9.12,11.3)$ & - & $0.989^{b}$ & $11.9-12.3 / 9.41-9.83$ \\
\hline \multicolumn{9}{|l|}{ Macronutrients } \\
\hline Carbohydrate (EN\%) & $46.7(43.6,49.8)$ & $0.824^{c}$ & $49.4(45.5,53.3)$ & $0.067^{c}$ & $55.2(51.4,59.0)$ & $0.002^{c}$ & $0.003^{\mathrm{a}}$ & $>50$ \\
\hline $\begin{array}{l}\text { Carbohydrate } \\
\text { (g/kg BW) }\end{array}$ & $4.31(3.45,5.17)$ & $1.000^{c}$ & $4.22(3.52,4.91)$ & $0.094^{c}$ & $5.01(4.40,5.62)$ & $0.111^{c}$ & $0.049^{b}$ & \\
\hline Protein (EN\%) & $16.7(15.1,18.9)$ & $0.540^{c}$ & $15.9(13.6,18.2)$ & $0.295^{c}$ & $13.8(12.5,15.0)$ & $0.007^{c}$ & $0.009^{b}$ & \\
\hline Protein (g/kg BW) & $1.50(1.27,1.66)$ & $0.159^{c}$ & $1.34(1.09,1.56)$ & $1.000^{c}$ & $1.25(1.07,1.42)$ & $0.063^{c}$ & $0.047^{b}$ & 0.8 \\
\hline Fat (EN\%) & $32.5(30.5,34.5)$ & $0.432^{c}$ & $30.8(26.8,34.8)$ & $0.708^{c}$ & $26.3(22.7,29.8)$ & $0.021^{c}$ & $0.026^{\mathrm{b}}$ & 30 \\
\hline Fiber (g) & $27.0(22.8,31.1)$ & $0.176^{\mathrm{c}}$ & $33.4(28.6,38.2)$ & $0.006^{c}$ & $51.7(44.1,59.4)$ & $0.000^{c}$ & $0.000^{b}$ & $\geq 30$ \\
\hline
\end{tabular}

OMN omnivores, LOV lacto-ovo vegetarians, VEG vegans, $M J$ mega joule, EN\% energy percent, $B W$ body weight, *Reference values of the German, Austrian and Swiss Nutrition Societies (Deutsche, Österreichische und Schweizerische Gesellschaften für Ernährung, D-A-CH) [33]. Data are presented as mean (95\% Cl). ${ }^{a}$ One-

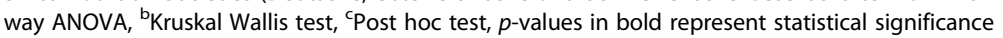

VEG, who were below the recommendation of $30 \mathrm{EN} \%$ (Table 3). A low-fat intake (<30 EN\%) was observed in $70.4 \%$ of the VEG, $44.0 \%$ of the LOV and $25.9 \%$ of the OMN group. These differences were significant $(p=0.004$, $x^{2}$ ). Differences in fatty acid intake patterns were observed. The highest intake of saturated fatty acids was observed in the OMN group (8.70, 7.13-10.3 EN\%) followed by LOV (7.86, 6.17-9.55 EN\%; n.s. compared to OMN) and VEG (4.57, 3.55-5.59 EN\%; POMN-VEG $<0.001$ ) (see Additional file 2). Monounsaturated fatty acids were least consumed by the VEG group (3.96, 3.02-4.91 EN\%) compared to LOV $(5.45,3.77-7.13$ EN\%; n.s. compared to LOV) and OMN (5.95, 4.86-7.03 EN\%; POMN-VEG = 0.019 ). No differences were observed in polyunsaturated fatty acid (PUFA) intake. On average, none of the three groups reached the recommended intake values of monounsaturated fatty acids (>10 EN\%) and PUFA (7-10 EN\%). The intake of linoleic acid (LA) was 4.33 (3.445.21) EN\% in the VEG group, $3.52(2.57-4.46) \mathrm{EN} \%$ in LOV and 2.96 (2.50-3.42) EN\% in OMN. Similarly, the intake of alpha-linolenic acid (ALA) was highest in the VEG group (0.80, 0.55-1.05 EN\%) compared to LOV (0.68, 0.33-1.03 EN\%, n.s. compared to VEG) and OMN (0.37, 0.27-0.48 EN\%, POMN-VEG $=0.005)$. The ratio LA:ALA did not differ significantly between the groups, although OMN showed a less favorable ratio (1:8.04) (see Additional file 2). The PUFAs, eicosapentaenoic acid (EPA, 20: $5 \mathrm{n} 3$ ) and docosahexaenoic acid (DHA, 22:6n3), were supplemented by two subjects of the OMN group, two subjects of the VEG and one of the LOV group. We observed the highest sum of EPA + DHA intake in the OMN group $(0.54,0.23-0.85 \mathrm{~g})$, followed by LOV $(0.08,0.37-0.12 \mathrm{~g}$; POMN-LOV $=0.003)$ and VEG $(0.09,0.01-0.17 \mathrm{~g}$; POMN-VEG $<0.001$ ).

Fiber intake was significantly higher in the VEG group (51.7, 44.1-59.4 g) compared to LOV (33.4, 28.6-38.2 g;
POMN-LOV $=0.006)$ and OMN $(27.0,22.8-31.1 \mathrm{~g}$; POMN-VEG $<0.001)$. The latter did not reach the minimum reference value of $30 \mathrm{~g}$ per day.

Micronutrient intakes also showed several differences between the groups (Table 4). Several participants did not reach the recommended intake for all the micronutrients examined (see Additional file 3). There were variations regarding the minerals sodium, potassium and magnesium, while calcium and phosphorus values were similar. More precisely, lower sodium intake was observed in LOV ( $\mathrm{p}_{\mathrm{OMN}-\mathrm{LOV}}=0.004$ ) and VEG ( $\mathrm{p}_{\mathrm{OMN}-\text { - }}$ $\mathrm{VEG}=0.005)$ compared to OMN (Table $4, p$ values of total intake are not shown). By contrast, the VEG group had significantly higher intake levels of potassium and magnesium compared to LOV ( $\mathrm{p}_{\text {LOV-VEG }}=0.005$ and 0.001 , respectively) and OMN ( $\mathrm{POMN}-\mathrm{VEG}=0.014$ and $<$ 0.001 , respectively) (Table $4, \mathrm{p}$ values of total intake are not shown). On average, the LOV and VEG groups had calcium intakes < $1000 \mathrm{mg}$ per day [33], and OMN consumed sufficient amounts $(1026,846-1207 \mathrm{mg})$ due to supplementation. A total of $64.0 \%$ of the LOV group, $51.9 \%$ of OMN and $44.4 \%$ of VEG were below the recommendations for calcium (see Additional file 3).

There were also group differences regarding trace elements, except for the zinc values, which did not vary between the groups. All three groups had adequate dietary zinc intakes, however, the male LOVs were slightly low $(9.89,5.33-14.5 \mathrm{mg})$. Female subjects reached the recommendations and so did the non-SU (OMN: 8.46, 6.30-10.6 mg; LOV: 9.44, 6.77-12.1 mg; VEG: 9.89, 7.63-12.1 mg). We observed a high iron intake, particularly in the VEG group (Table 4). The mean iron intake was within the recommended area $(10 \mathrm{mg} /$ day [33]) in all three groups when only men were compared, and in both male SU and non-SU (Fig. 2). The highest iron intake via food in women was found in the VEG group 
Table 4 Dietary mineral intake of the study population calculated from a 3-day dietary record (nutrient intake via food and supplements)

\begin{tabular}{|c|c|c|c|c|c|c|c|c|}
\hline & $\begin{array}{l}\text { OMN } \\
(n=27)\end{array}$ & $\begin{array}{l}p \text { value } \\
\text { OMN-LOV }\end{array}$ & $\begin{array}{l}\text { LOV } \\
(n=25)\end{array}$ & $\begin{array}{l}p \text { value } \\
\text { LOV-VEG }\end{array}$ & $\begin{array}{l}\text { VEG } \\
(n=27)\end{array}$ & $\begin{array}{l}p \text { value } \\
\text { OMN-VEG }\end{array}$ & $\begin{array}{l}p \text { value } \\
3 \text { groups }\end{array}$ & Reference values $(\mathrm{m} / \mathrm{f})^{*}$ \\
\hline \multicolumn{9}{|l|}{$\mathrm{Na}(\mathrm{g})$} \\
\hline Food & $2.65(2.17,3.12)$ & $0.004^{b}$ & $1.72(1.44,2.00)$ & $1.000^{\mathrm{b}}$ & $1.72(1.46,1.99)$ & $0.005^{b}$ & $0.001^{\mathrm{a}}$ & 1.5 \\
\hline Supplement & 0 & - & 0 & - & 0 & - & - & \\
\hline \multicolumn{9}{|l|}{ K (g) } \\
\hline Food & $3.16(2.88,3.50)$ & $1.000^{\mathrm{b}}$ & $3.04(2.55,3.52)$ & $0.005^{b}$ & $4.65(3.85,5.50)$ & $0.014^{b}$ & $0.002^{\mathrm{a}}$ & $4^{c}$ \\
\hline Supplement & 0 & - & $0.00(0.00,0.01)$ & - & $0.00(0.00,0.01)$ & - & $0.372^{a}$ & \\
\hline \multicolumn{9}{|l|}{$\mathrm{Ca}(\mathrm{mg})$} \\
\hline Food & $981(813,1149)$ & - & $901(716,1085)$ & - & $730(614,846)$ & - & $0.115^{\mathrm{a}}$ & 1000 \\
\hline Supplement & $45.1(-32.0,122)$ & - & 0 & - & $6.37(-2.22,15.0)$ & - & $0.214^{\mathrm{a}}$ & \\
\hline \multicolumn{9}{|l|}{$P(g)$} \\
\hline Food & $1.43(1.26,1.60)$ & - & $1.34(1.08,1.61)$ & - & $1.33(1.15,1.52)$ & - & $0.495^{a}$ & 0.7 \\
\hline Supplement & 0 & - & 0 & - & 0 & - & - & \\
\hline \multicolumn{9}{|l|}{$\mathrm{Mg}(\mathrm{mg})$} \\
\hline Food & $346(310,382)$ & $0.990^{\mathrm{b}}$ & $388(324,452)$ & $0.001^{\mathrm{b}}$ & $599(518,679)$ & $0.000^{b}$ & $0.000^{\mathrm{a}}$ & $350 / 300$ \\
\hline Supplement & $36.7(0.44,73.0)$ & - & $53.2(-5.58,112)$ & - & $54.3(-7.09,116)$ & - & $0.910^{\mathrm{a}}$ & \\
\hline \multicolumn{9}{|l|}{$\mathrm{Fe}(\mathrm{mg})$} \\
\hline Food (total) & $11.9(10.6,13.2)$ & $1.000^{\mathrm{b}}$ & $12.8(10.8,14.7)$ & $0.001^{b}$ & $19.6(16.8,22.4)$ & $0.000^{b}$ & $0.000^{\mathrm{a}}$ & $10 / 15$ \\
\hline Plant-based iron & $7.44(6.33,8.54)$ & $0.105^{b}$ & $10.7(8.95,12.5)$ & $0.000^{\mathrm{b}}$ & $19.6(16.8,22.4)$ & $0.000^{\mathrm{b}}$ & $0.000^{\mathrm{a}}$ & \\
\hline Animal iron & $4.45(3.67,5.24)$ & $0.013^{b}$ & $2.02(1.41,2.61)$ & $0.000^{b}$ & 0 & $0.000^{\mathrm{b}}$ & $0.000^{\mathrm{a}}$ & \\
\hline Supplement & $1.70(-1.36,4.77)$ & - & $1.52(-1.19,4.24)$ & - & $3.74(-0.64,8.12)$ & - & $0.675^{a}$ & \\
\hline \multicolumn{9}{|l|}{$\mathrm{Zn}(\mathrm{mg})$} \\
\hline Food & $9.74(8.32,11.2)$ & - & $8.88(7.30,10.5)$ & - & $10.7(9.21,12.2)$ & - & $0.214^{\mathrm{a}}$ & $14 / 8^{1}$ \\
\hline Supplement & $2.23(-1.59-6.04)$ & - & $0.90(-0.70-2.49)$ & - & $0.47(-0.48-1.41)$ & - & $0.648^{a}$ & \\
\hline \multicolumn{9}{|l|}{$\mathrm{Cu}(\mathrm{mg})$} \\
\hline Food & $1.63(1.43,1.84)$ & $0.819^{b}$ & $1.85(1.56,2.13)$ & $0.001^{b}$ & $2.93(2.51,3.34)$ & $0.000^{b}$ & $0.000^{\mathrm{a}}$ & $1.0-1.5$ \\
\hline Supplement & 0 & - & 0 & - & 0 & - & - & \\
\hline \multicolumn{9}{|l|}{$\mathrm{Mn}(\mathrm{mg})$} \\
\hline Food & $4.75(3.87,5.62)$ & $0.188^{b}$ & $6.29(5.05,7.54)$ & $0.067^{b}$ & $8.48(7.10,9.85)$ & $0.000^{\mathrm{b}}$ & $0.000^{\mathrm{a}}$ & $2.0-5.0$ \\
\hline Supplement & 0 & - & 0 & - & 0 & - & - & \\
\hline \multicolumn{9}{|l|}{ I $(\mu \mathrm{g})$} \\
\hline Food & $88.8(64.1,114)$ & $0.190^{\mathrm{b}}$ & $61.6(49.4,73.7)$ & $1.000^{\mathrm{b}}$ & $57.7(48.4,67.0)$ & $0.060^{\mathrm{b}}$ & $0.048^{\mathrm{a}}$ & 200 \\
\hline Supplement & 0 & - & 0 & - & 0 & - & - & \\
\hline
\end{tabular}

OMN omnivores, LOV lacto-ovo vegetarians, VEG vegans, *Reference values of the German, Austrian and Swiss Nutrition Societies (Deutsche, Österreichische und Schweizerische Gesellschaften für Ernährung, D-A-CH) [33]

Data are presented as mean $(95 \% \mathrm{Cl}) .{ }^{a}$ Kruskal Wallis test, ${ }^{\mathrm{b}}$ Post hoc test, ${ }^{\mathrm{c}}$ Estimated values, $p$-values in bold represent statistical significance ${ }^{1}$ At medium phytate intake

$(19.8, \quad 15.7-24.0 \mu \mathrm{g})$, followed by $\operatorname{LOV}(12.8,9.47-$ $\left.16.1 \mu \mathrm{g} ; \mathrm{p}_{\mathrm{LOV}-\mathrm{VEG}}=0.037\right)$ and OMN $(11.2,9.01-13.2 \mu \mathrm{g}$; POMN-VEG $=0.005)$. Only the female SU in both the LOV and OMN groups reached the reference range $(15 \mathrm{mg} /$ day [33]) (Fig. 3). The iron sources in the diet of the VEG group were exclusively plant-based food. However, the LOV and OMN groups consumed predominantly plantbased iron as well (Table 4). The worst supply was observed for iodine. Only $3.7 \%$ of the OMN group and none of the subjects in LOV and VEG had values in a reference range of $200 \mu \mathrm{g}$ per day (see Additional file 3) [33].

Variations were also observed in the vitamin intake between the groups (Table 5). On average, all three groups reached the recommended amounts for thiamine, pyridoxine and folate, while the reference value for vitamin $\mathrm{D}$ was not achieved, and the ascorbic acid intake was exceeded in all groups. Due to the supplementation, the highest average intake of cobalamin was observed in the 
Table 5 Dietary vitamin intake of the study population calculated from a 3-day dietary record (nutrient intake via food and supplements)

\begin{tabular}{|c|c|c|c|c|c|c|c|c|c|}
\hline & & $\begin{array}{l}\text { OMN } \\
(n=27)\end{array}$ & $\begin{array}{l}p \text { value } \\
\text { OMN-LOV }\end{array}$ & $\begin{array}{l}\text { LOV } \\
(n=25)\end{array}$ & $\begin{array}{l}p \text { value } \\
\text { LOV-VEG }\end{array}$ & $\begin{array}{l}\text { VEG } \\
(n=27)\end{array}$ & $\begin{array}{l}p \text { value } \\
\text { OMN-VEG }\end{array}$ & $\begin{array}{l}p \text { value } \\
3 \text { groups }\end{array}$ & $\begin{array}{l}\text { Reference values } \\
(\mathrm{m} / \mathrm{f})^{*}\end{array}$ \\
\hline \multirow[t]{2}{*}{$\begin{array}{l}\text { A [retinol equ.] } \\
\text { (mg) }\end{array}$} & Food & $\begin{array}{l}1.45 \\
(0.81,2.10)\end{array}$ & - & $\begin{array}{l}1.26 \\
(0.91,1.61)\end{array}$ & - & $\begin{array}{l}1.72 \\
(1.27,2.16)\end{array}$ & - & $0.221^{a}$ & $1.0 / 0.8$ \\
\hline & Supplement & 0 & - & 0 & - & 0 & - & - & \\
\hline \multirow[t]{2}{*}{$D(\mu g)$} & Food & $\begin{array}{l}2.61 \\
(1.34,3.89)\end{array}$ & $1.000^{\mathrm{b}}$ & $\begin{array}{l}1.67(1.02, \\
2.32)\end{array}$ & $0.037^{b}$ & $\begin{array}{l}1.04 \\
(0.46,1.62)\end{array}$ & $0.003^{b}$ & $0.002^{\mathrm{a}}$ & 20 \\
\hline & Supplement & $\begin{array}{l}5.68 \\
(-0.12,11.5)\end{array}$ & - & $\begin{array}{l}2.75 \\
(-2.91,8.40)\end{array}$ & - & $\begin{array}{l}18.8 \\
(1.61,36.1)\end{array}$ & - & $0.086^{\mathrm{a}}$ & \\
\hline \multirow[t]{2}{*}{$\mathrm{E}(\mathrm{mg})$} & Food & $\begin{array}{l}9.66 \\
(7.85,11.5)\end{array}$ & $0.851^{\mathrm{b}}$ & $\begin{array}{l}11.4 \\
(9.03,13.7)\end{array}$ & $0.280^{\mathrm{b}}$ & $\begin{array}{l}16.4 \\
(12.5,20.4)\end{array}$ & $0.015^{b}$ & $0.018^{a}$ & $14 / 12^{c}$ \\
\hline & Supplement & $\begin{array}{l}1.12 \\
(-0.47,2.71)\end{array}$ & - & $\begin{array}{l}0.15 \\
(-0.16,0.47)\end{array}$ & - & $\begin{array}{l}0.04 \\
(-0.37,0.11)\end{array}$ & - & $0.411^{\mathrm{a}}$ & \\
\hline \multirow[t]{2}{*}{$K(\mu \mathrm{g})$} & Food & $\begin{array}{l}92.5 \\
(63.5,122)\end{array}$ & $0.119^{b}$ & $181(96.6,266)$ & $0.058^{b}$ & $261(164,359)$ & $0.000^{\mathrm{b}}$ & $0.000^{a}$ & $70 / 60$ \\
\hline & Supplement & 0 & - & 0 & - & 0 & - & - & \\
\hline \multirow[t]{2}{*}{$\mathrm{B}_{1}$ [thiamine] (mg) } & Food & $\begin{array}{l}1.38 \\
(1.21,1.55)\end{array}$ & $0.502^{\mathrm{b}}$ & $\begin{array}{l}1.20(0.98, \\
1.43)\end{array}$ & $0.003^{b}$ & $\begin{array}{l}1.86 \\
(1.56,2.16)\end{array}$ & $0.143^{b}$ & $0.004^{a}$ & $1.2 / 1.0$ \\
\hline & Supplement & $\begin{array}{l}0.56 \\
(-0.58,1.70)\end{array}$ & - & $\begin{array}{l}0.17 \\
(-0.16,0.50)\end{array}$ & - & $\begin{array}{l}0.09 \\
(-0.08,0.26)\end{array}$ & - & $0.888^{a}$ & \\
\hline \multirow[t]{2}{*}{$\begin{array}{l}B_{2} \text { [riboflavin] } \\
\text { (mg) }\end{array}$} & Food & $\begin{array}{l}1.57 \\
(1.34,1.79)\end{array}$ & - & $\begin{array}{l}1.54 \\
(1.12,1.96)\end{array}$ & - & $\begin{array}{l}1.38 \\
(1.16,1.59)\end{array}$ & - & $0.278^{a}$ & $1.4 / 1.1$ \\
\hline & Supplement & $\begin{array}{l}0.56 \\
(-0.58,1.70)\end{array}$ & - & $\begin{array}{l}0.01 \\
(-0.01,0.03)\end{array}$ & - & $\begin{array}{l}0.11 \\
(-0.98,0.33)\end{array}$ & - & $0.896^{\mathrm{a}}$ & \\
\hline \multirow[t]{2}{*}{ Niacin (mg) } & Food & $21.4(18.5,24.3)$ & $0.033^{b}$ & $\begin{array}{l}15.8 \\
(12.3,19.3)\end{array}$ & $1.000^{\mathrm{b}}$ & $\begin{array}{l}17.3 \\
(12.3,22.3)\end{array}$ & $0.021^{b}$ & $0.010^{a}$ & $15 / 12$ \\
\hline & Supplement & $0.62(-0.52,1.77)$ & - & $\begin{array}{l}0.09 \\
(-0.09,0.27)\end{array}$ & - & $\begin{array}{l}1.31 \\
(-1.12,3.74)\end{array}$ & - & $0.645^{\mathrm{a}}$ & \\
\hline \multirow[t]{2}{*}{$\begin{array}{l}\text { Pantothenic acid } \\
\text { (mg) }\end{array}$} & Food & $5.23(4.38,6.07)$ & - & $\begin{array}{l}5.36 \\
(4.04,6.68)\end{array}$ & - & $\begin{array}{l}6.39 \\
(4.96,7.81)\end{array}$ & - & $0.461^{a}$ & $6^{c}$ \\
\hline & Supplement & $\begin{array}{l}0.95(-0.95 \\
2.85)\end{array}$ & - & 0 & - & $\begin{array}{l}0.04 \\
(-0.19,0.11)\end{array}$ & - & $0.374^{a}$ & \\
\hline \multirow[t]{2}{*}{$\begin{array}{l}\text { B6 [pyridoxine] } \\
\text { (mg) }\end{array}$} & Food & $1.91(1.61,2.20)$ & $0.670^{\mathrm{b}}$ & $\begin{array}{l}1.59 \\
(1.27,1.91)\end{array}$ & $0.002^{b}$ & $\begin{array}{l}2.63 \\
(2.10,3.16)\end{array}$ & $0.087^{b}$ & $0.003^{a}$ & $1.6 / 1.4$ \\
\hline & Supplement & $\begin{array}{l}0.47 \\
(-0.31,1.25)\end{array}$ & - & $\begin{array}{l}0.46 \\
(-0.11,1.04)\end{array}$ & - & $\begin{array}{l}0.16(-0.07 \\
0.40)\end{array}$ & - & $0.497^{\mathrm{a}}$ & \\
\hline \multirow[t]{2}{*}{ Biotin $(\mu \mathrm{g})$} & Food & $50.9(44.9,56.9)$ & - & $\begin{array}{l}56.7 \\
(43.4,69.9)\end{array}$ & - & $\begin{array}{l}64.5 \\
(51.4,77.6)\end{array}$ & - & $0.573^{a}$ & $30-60^{c}$ \\
\hline & Supplement & $6.10(-5.33,17.5)$ & - & 0 & - & $\begin{array}{l}0.70 \\
(-0.44,1.90)\end{array}$ & - & $0.373^{a}$ & \\
\hline \multirow[t]{2}{*}{ Folate $(\mu \mathrm{g})$} & Food & $307(249,364)$ & $1.000^{\mathrm{b}}$ & $327(265,389)$ & $0.024^{b}$ & $478(402,572)$ & $0.001^{b}$ & $0.001^{\mathrm{a}}$ & 300 \\
\hline & Supplement & $11.3(-5.01,27.6)$ & - & $\begin{array}{l}2.20 \\
(-2.33,6.72)\end{array}$ & - & $\begin{array}{l}41.9 \\
(-20.2,104)\end{array}$ & - & $0.261^{a}$ & \\
\hline \multirow[t]{2}{*}{$\begin{array}{l}\text { B }_{12} \text { [cobalamin] } \\
(\mu \mathrm{g})\end{array}$} & Food & $4.02(3.12,4.92)$ & $0.057^{c}$ & $\begin{array}{l}2.49(1.49 \\
3.48)\end{array}$ & $0.002^{b}$ & $\begin{array}{l}0.79 \\
(0.47,1.12)\end{array}$ & $0.000^{b}$ & $0.000^{a}$ & 4 \\
\hline & Supplement & $0.96(-0.21,2.13)$ & $0.002^{b}$ & $\begin{array}{l}0.84 \\
(-0.20,1.89)\end{array}$ & $1.000^{b}$ & $206(101,312)$ & $0.004^{b}$ & $0.001^{\mathrm{a}}$ & \\
\hline \multirow{2}{*}{$\begin{array}{l}\text { C [ascorbic acid] } \\
\text { (mg) }\end{array}$} & Food & $153(110,196)$ & $1.000^{\mathrm{b}}$ & $143(107,179)$ & $0.003^{b}$ & $293(222,365)$ & $0.001^{b}$ & $0.000^{a}$ & $110 / 95$ \\
\hline & Supplement & $3.16(-1.07,7.38)$ & - & $\begin{array}{l}0.17 \\
(-0.18,0.51)\end{array}$ & - & $\begin{array}{l}7.80 \\
(-1.26,13.7)\end{array}$ & - & $0.126^{\mathrm{a}}$ & \\
\hline
\end{tabular}


VEG group $(207,102-313 \mu \mathrm{g})$, followed by OMN (4.97, 3.70-6.25 $\mu$ g; n.s. compared to VEG) and LOV (2.96, 1.69-4.24 $\mu$ g; n.s. compared to VEG) (Table 5). Riboflavin intake was low in $44.4 \%$ of VEG subjects, $44.0 \%$ of LOV and $22.2 \%$ of OMN (see Additional file 3). We found the highest vitamin D intake in the VEG group $(19.9,2.75-37.0 \mu \mathrm{g})$, followed by $\operatorname{OMN}(8.29,2.22-$ $14.37 \mu$ g; n.s. compared to VEG) and LOV (4.52, - 1.34$10.39 \mu$ g; n.s. compared to VEG) (Table 5). Only $22.2 \%$ of the VEG group, $14.8 \%$ of OMN and $4.00 \%$ of LOV had vitamin $\mathrm{D}$ intakes within the recommendations $(20 \mu \mathrm{g} /$ day [33]).

\section{Discussion}

Organizations such as The American College of Sports Medicine (ACSM), The International Society for Sports Nutrition (ISSN) and the International Olympic Committee (IOC) have defined guidelines for athletes [35-37]. As these few existing recommendations for mainly high-performance athletes were only partially applicable to this study collective, the nutrient intake was compared with intake recommendations of the D-A-CH for the general population. However, the $\mathrm{D}-\mathrm{A}-\mathrm{CH}$ does not specify any certain reference values for ambitious recreational athletes [33].

In general, recreational athletes can be supplied with all micronutrients through a balanced mixed diet. But, it is unknown whether a vegetarian and especially vegan diet can provide all the important nutrients for athletes.

The type, duration and intensity of sport determines the energy requirements. The ISSN recommends an energy intake from $7.5-10.0 \mathrm{MJ}(1800-2400 \mathrm{kcal})$ for athletes with general physical activity levels of $30-40 \mathrm{~min}$ three to four times a week [35]. In order to assess the energy demand, the ACSM recommends various options (e.g. based on the daily recommended intake, the basal metabolic rate and a factor of physical activity or metabolic equivalents) [37]. The IOC refers to the fat-free mass (30-45 kcal/kg FFM/day) [38]. Our subjects trained an average of three times a week for about $60 \mathrm{~min}$, which corresponds to an estimated physical activity level value of about 1.7 (sedentary work and recreationally active) [33]. More than half of each group did not reach the recommended energy intake, which is not uncommon in endurance athletes [39]. There were no differences among the groups, which agrees with the results of Lynch and colleagues, who compared 35 vegetarian athletes with 35 omnivores [26].

\section{Macronutrients}

Carbohydrates are the most important sources of energy and many endurance athletes strive to consume carbohydrates to benefit from full glycogen stores [40]. Depending on the intensity and type of training or competition, gender, and external influences, an absolute amount of $3-7 \mathrm{~g} / \mathrm{kg} \mathrm{BW}$ is recommended for people with general physical activity of about 30-60 min/day 34 times a week up to about 1 hour a day [35-37]. Thus, participants in the present study achieved the recommendations for carbohydrate intake [35-37]. Similar to previous studies with non-athletes [41-44], the VEG group had the highest intake of carbohydrates (55.2, 51.4-59.0 EN\%) compared to OMN (46.7, 43.6-49.8 EN\%; POMN-VEG $=0.002)$ and LOV $(49.4,45.5-53.3$ EN\%; n.s. compared to VEG), which can be explained by the increased intake of potatoes and fruit, since the intake of whole-grain and cereal products, pastries and sweets were similar for all groups.

The protein needs of athletes have been widely discussed [45-47]. The three societies recommend a range of $1.2-2.0 \mathrm{~g} / \mathrm{kg} \mathrm{BW}$ for most exercising individuals (including general fitness [35]) [35-37]. According to the IOC and ACSM, the recommended amount also applies to vegetarians. The average protein intake of all three groups was within the reference range. In addition to absolute protein intake, it is important to consider the quality of the proteins [35]. Protein sources were mainly meat, meat products and sausages, fish and dairy products for the OMN group, milk, dairy products, and eggs for LOV, and cereal products, legumes and soybeans for VEG. In general, a high biological value can be achieved with each of these three diets. Compared to the reference values of the WHO, on average, all groups met the reference range for amino acid intake [34]. Hence, it can be assumed that all three groups - including VEG - had an adequate protein and amino acid supply. Our findings are consistent with the literature, which has shown that non-athlete LOV and VEG appear to be within the range of recommendations for protein intake $[44,48]$.

Dietary fats are valuable energy sources and have structural and regulatory functions. Dietary recommendations for adequate fat intakes vary widely and depend on the level of training and body composition goals [35-37]. While the ACSM recommends a daily intake of 20-35 $\mathrm{EN} \%$ but not less than $20 \mathrm{EN} \%$ fat [37], the IOC advises an intake of $\geq 15-20$ EN\% fat, depending on the type of sport [49]. By contrast, both D-A-CH and ISSN recommend a fat intake of $30 \mathrm{EN} \%[33,35]$. Most subjects in the three groups reached the recommendations of the D-A$\mathrm{CH}$ [33], ISSN and ACSM. In addition, it is important to evaluate the PUFA intake of athletes, which was below the reference value in all three groups [33]. PUFAs play a pivotal role in health due to their precursor function as regulatory lipid mediators. The International Society for the Study of Fatty Acids and Lipids recommends a daily sum EPA + DHA intake of $0.5 \mathrm{~g}$, which was achieved by the OMN group (0.54, $0.23-0.85 \mathrm{~g})$, but not by LOV $(0.08$, 0.04-0.12 g; POMN-LOV $=0.003)$ or VEG $(0.09,0.01-0.17 \mathrm{~g}$; POMN-VEG $<0.001)$ [50]. PUFA intakes in LOV and VEG 
within this study can be classified as inadequate, which is consistent with other studies regarding non-athlete vegetarians and vegans [51]. The EPA/DHA supplements were only consumed occasionally in the VEG and LOV groups. The resulting LA:ALA ratios in the VEG (1:5.71) and LOV groups (1:5.30) were within the reference range [33]. The OMN group showed higher LA:ALA ratios (1:8.04), which are consistent with the results of the German $\mathrm{Nu}$ trition Survey [52].

\section{Micronutrients}

It is generally thought that athletes consume high amounts of micronutrients via dietary supplements due to their increased health awareness [53]. However, several studies have shown insufficient micronutrient intake in athletes $[54,55]$. There are no specific recommendations for micronutrient intakes in recreationally active individuals, which differ from the general population's guidelines. However, in the view of the ACSM, ISSN and IOC, an adequate supply of micronutrients is assured with a balanced mixed diet. A possible insufficient supply to vegetarians of zinc, iron, riboflavin, cobalamin and vitamin D is described in the ACSM and IOC guidelines [36, 37], while the ACSM additionally mentions calcium, pyridoxine and folate. A specific risk of an insufficient micronutrient supply with a vegan diet is not mentioned.

In the present study, magnesium, calcium, iron, vitamin $\mathrm{D}$ and cobalamin were the most frequently supplemented nutrients. Cobalamin intake was strongly dependent on supplementation, especially for both female and male VEG. Half of the VEG group supplemented cobalamin and, thus, had a significantly higher intake compared to the D-A-CH reference values of $4 \mu \mathrm{g}$ per day [33]. However, the very high cobalamin intake of the vegan supplement user can be classified as uncritical [56, 57]. In addition, the absorption rate decreases with increasing dosage. As expected, subjects of the VEG group who did not take cobalamin supplements had a marginal intake. Additionally, the dietary intake of the LOV group was insufficient, especially for males, who had cobalamin intakes below the recommendations, regardless of supplementation. However, although consuming cobalamin-rich foods such as meat, meat products and fish, its intake was still inadequate in one-third of the OMN group. Cobalamin is considered critical for VEG, but adequate intake should be ensured for every diet.

Due to high riboflavin levels in animal products, it was not surprising that the OMN group consumed the highest amounts, although, on average, VEG and female LOV reached the recommendations, which agrees with previous studies in non-athletes $[58,59]$. In contrast to Eisinger and colleagues, who showed high intakes of riboflavin in LOV endurance runners [60], only female
LOV achieved the reference values. Pyridoxine intake exceeded the recommendations in the VEG group due to the high consumption of vegetables, legumes, nuts, and seeds, which has already been shown by other studies with non-athletes [58, 61]. The VEG group showed a high folate intake due to the high amount of folate in green vegetables, yeast, and nuts, while the folate intake of most OMN subjects was insufficient. These results are consistent with the German Nutrition Survey [52] and studies with athletes [54].

Similar to cobalamin, vitamin D intake was strongly dependent on the use of supplements. This becomes clear by comparing the vitamin D intake between SU and non-SU. On average, the VEG group (19.9, 2.75$37.0 \mu \mathrm{g})$ was closest to the recommendations of $20 \mu \mathrm{g}$ per day compared to $\operatorname{OMN}(8.29,2.21-14.4 \mu \mathrm{g})$ and LOV $(4.52,-1.14-10.4 \mu \mathrm{g})$. However, the intake of vitamin D was considerably higher in SU compared to nonSU. Hence, the mean values for the vitamin D intake in the VEG group (including SU and non-SU) should be treated with caution. This also applies to the OMN and LOV group, although not quite as strongly pronounced. However, it is worth mentioning that an adequate vitamin D status can only be evaluated with the endogenous 25-hydroxyvitamin D status in the blood [62].

Similar to other studies with non-athletes $[42,58]$, the highest iron intake from food (excluding supplements) was observed in VEG subjects compared to LOV and OMN. In addition, the VEG group had the highest iron intake via supplements compared to the other two groups. A total of more than $85 \%$ of VEG subjects achieved the recommendations compared to only 50\% in OMN and LOV. Male subjects of all groups were above the recommendations with more than $10 \mathrm{mg}$ per day, independent of supplementation. Female OMN and LOV subjects achieved the recommendation of $15 \mathrm{mg}$ daily only after supplementation. Interestingly, the VEG group reached the iron intake recommendations solely via food and not via supplements. The literature on the iron supply of athletes is inconsistent. Some studies found an adequate $[63,64]$ and others an inadequate iron intake in athletes [65]. High-performance athletes might have increased requirements due to biochemical adaptations (e.g. increased blood formation and increased enzymatic antioxidant defense) and increased iron losses via sweat, urine, and feces, which results in a higher risk of iron deficiency anemia [64]. In addition to absolute amounts, the bioavailability of different iron species should be considered. Despite the exclusive consumption of plant-based iron of the VEG group, LOV and $\mathrm{OMN}$ also consumed predominantly plant iron sources. While plant-based foods contain non-heme iron, mainly in trivalent form $\left(\mathrm{Fe}^{3+}\right)$, which has a poor bioavailability of $1-5 \%$, meat and fish contain about $70 \%$ 
of the total iron in the form of heme iron, which can be absorbed much better at $10-20 \%[66,67]$. Hence, the lower iron intake in OMN subjects compared to LOV and VEG does not necessarily result in a lower status. Moreover, further influences on bioavailability (promoting substances such as ascorbic acid or lactic acid and inhibiting substances such as phytic acid or oxalic acid, which occurs in vegetable foods) must be taken into account (the same applies to zinc, magnesium, and calcium). Therefore, only functional parameters, such as transferrin and ferritin, indicate an adequate supply status.

The present results show that calcium is a critical nutrient [55]. As expected, calcium intake was highest among OMN subjects, although more than half were below the reference range. The highest number of subjects with an intake below the reference range for calcium was found in the LOV group (64.0\%), although they consumed milk and dairy products. The athlete's dietary intake of calcium should be improved independently of dietary habits due to the importance of bone health, and normal nerve and muscle function [68]. The mean intake of zinc was within the reference range for all groups, although male LOV subjects were slightly below. Female participants and non-SU of all three groups reached the recommendations [33]. Interestingly, the zinc supply was similar in OMN and VEG subjects, although animal-based foods are rich in zinc and the zinc supplement intake in the VEG group was considerably lower than OMN. These results reveal that zincrich plant-based foods can secure adequate zinc supply. The literature on zinc supply is inconsistent. Some studies observed a slightly lower but adequate intake of zinc in vegetarians and VEG compared to $\mathrm{OMN}[43,48,58]$, other studies found no differences between vegetarian and OMN endurance athletes [26].

The fact that the data of dietary intake relied on selfreported data by subjects should be considered. Both under- and over-reporting are further sources of error in dietary records. Since the use of iodized salt is voluntary in Germany and a precise indication about the dietary intake is critical, the values of iodine intake should be considered with caution. Furthermore, there are limitations regarding the nutrition software that shows data gaps, especially regarding vegan products. We did not consider the water intake of the subjects, which might also influence nutrient (e.g. mineral) supply.

\section{Conclusion}

In summary, all three groups were adequately supplied with most nutrients. As expected, the intake of carbohydrates and fiber was highest in the VEG group, while the recommended amount of fat was not reached. Moreover, all three groups exceeded the recommendations for absolute protein intake. The mean intake of micronutrients was partly dependent on supplementation, especially for vitamin D and cobalamin. Only female VEG achieved the recommended amounts for iron intake solely via food and not via supplements. However, the demand for several micronutrients might be higher for athletes due to increased requirements and losses, especially when exogenous factors such heat occur [69-72]. Recommendations of current guidelines for adequate micronutrient intakes of ambitious recreational athletes are sparse due to a lack of data and future studies should clarify if specific recommendations are necessary.

\section{Supplementary information}

Supplementary information accompanies this paper at https://doi.org/10 1186/s40795-019-0313-8 .

Additional file 1. Dietary intake of essential amino acids ( $\mathrm{mg} / \mathrm{kg} \mathrm{BW}$ ) according to dietary pattern.

Additional file 2. Dietary intake of fatty acids according to dietary pattern.

Additional file 3. Proportion of participants who did not reach the recommended dietary intake of minerals and vitamins. Dietary intake is depicted in addition to supplement intake.

\section{Abbreviations}

ACSM: American College of Sports Medicine; ALA: Alpha-linolenic acid; ANOVA: Analysis of variance; BMl: Body mass index; bpm: Beats per minute; BW: Body weight; Cl: Confidence interval; D-A-CH: Deutsche, Österreichische und Schweizerische Gesellschaften für Ernährung, German, Austrian and Swiss Nutrition Societies; DHA: Docosahexaenoic acid; EN\%: Energy percent; EPA: Eicosapentaenoic acid; IOC: International Olympic Committee; ISSN: International Society for Sports Nutrition; LA: Linoleic acid; LOV: Lactoovo vegetarians; n.S.: Not significant; non-SU: Non-supplement users; OMN: Omnivores; PUFA: Polyunsaturated fatty acid; SD: Standard deviation; SU: Supplement users; VEG: Vegans; WHO: World Health Organization

\section{Acknowledgments}

We would like to thank the participants who contributed their time to this study. The publication of this article was funded by the Open Access Fund of the Leibniz Universität Hannover.

\section{Authors' contributions}

JN, SH, UT and AH co-designed the study and survey materials. JN, PW, JE and $\mathrm{SH}$ were responsible for data acquisition. JN drafted the manuscript and conducted the statistical analyses. JPS and AH revised the manuscript critically for important content. All authors provided critical revisions and read and approved the final manuscript.

\section{Funding}

The study was funded by our own resources.

\section{Availability of data and materials}

The datasets used and/or analyzed during the current study are available from the corresponding author on reasonable request.

Ethics approval and consent to participate

The Ethics Committee at the Medical Chamber of Lower Saxony (Hannover, Germany) granted ethical approval for this research. The study was conducted in accordance with the Declaration of Helsinki. All participants gave their written informed consent.

Consent for publication

Not applicable. 


\section{Competing interests}

The authors declare that they have no competing interests.

\section{Author details}

${ }^{1}$ Faculty of Natural Sciences, Institute of Food Science and Human Nutrition, Leibniz University Hannover, 30167 Hannover, Germany. ${ }^{2}$ Hannover Medical School, Institute of Sports Medicine, 30625 Hannover, Germany.

Received: 1 February 2019 Accepted: 14 October 2019

Published online: 03 December 2019

\section{References}

1. Ströhle A, Löser C, Behrendt I, Leitzmann C, Hahn A. Alternative Ernährungsformen. Aktuel Ernährungsmed. 2016;41:47-65. https://doi.org/ 10.1055/s-0041-111459.

2. Ströhle $A$, Behrendt I, Behrendt $P$, Hahn A. Alternative Ernährungsformen Aktuel Ernährungsmed. 2016;41:120-38. https://doi.org/10.1055/s-0042-102322.

3. SKOPOS. 1,3 Millionen Deutsche leben vegan. 2016. Available from: https:// www.skopos.de/news/13-millionen-deutsche-leben-vegan.html (Accessed 13 Feb 2018)

4. Mensink GBM, Barbosa CL, Brettschneider A-K. Verbreitung der vegetarischen Ernährungsweise in Deutschland. J Health Monitor. 2016;2(1): 2-15. https://doi.org/10.17886/RKI-GBE-2016-033.

5. Allensbach I. Anzahl der Personen in Deutschland, die sich selbst als Vegetarier einordnen oder als Leute, die weitgehend auf Fleisch verzichten*, von 2007 bis 2018 (in Millionen): Statista; n.d. Available from: https://de. statista.com/statistik/daten/studie/173636/umfrage/lebenseinstellunganzahl-vegetarier/ (Accessed 5 Apr 2019)

6. WorldAtlas. Länder mit dem höchsten Anteil von Vegetariern an der Bevölkerung weltweit (Stand: 2016): Statista; 2016. Available from: https://de. statista.com/statistik/daten/studie/261627/umfrage/anteil-von-vegetariernund-veganern-an-der-bevoelkerung-ausgewaehlter-laender-weltweit/ (Accessed 5 June 2018)

7. Gallup. Few Americans Vegetarian or Vegan. 2018. Available from: https:// news.gallup.com/poll/238328/snapshot-few-americans-vegetarian-vegan. aspx (Accessed 5 Apr 2019).

8. Melina V, Craig W, Levin S. Position of the academy of nutrition and dietetics: vegetarian diets. J Acad Nutr Diet. 2016;116:1970-80. https://doi. org/10.1016/j.jand.2016.09.025.

9. Hever J. Plant-based diets: a physician's guide. Perm J. 2016;20(3):15-082 https://doi.org/10.7812/TPP/15-082.

10. Appleby PN, Key TJ. The long-term health of vegetarians and vegans. Proc Nutr Soc. 2016;75:287-93. https://doi.org/10.1017/S0029665115004334

11. Key TJ, Appleby PN, Spencer EA, Travis RC, Roddam AW, Allen NE. Cancer incidence in vegetarians: results from the European prospective investigation into Cancer and nutrition (EPIC-Oxford). Am J Clin Nutr. 2009; 89:1620S-6S. https://doi.org/10.3945/ajen.2009.26736M

12. Orlich MJ, Singh PN, Sabaté J, Jaceldo-Siegl K, Fan J, Knutsen S, et al. Vegetarian dietary patterns and mortality in Adventist health study 2. JAMA Intern Med. 2013;173:1230. https://doi.org/10.1001/jamainternmed. 2013.6473.

13. Pettersen BJ, Anousheh R, Fan J, Jaceldo-Siegl K, Fraser GE. Vegetarian diets and blood pressure among white subjects: results from the Adventist health Study-2 (AHS-2). Public Health Nutr. 2012;15:1909-16. https://doi.org/10. 1017/S1368980011003454

14. Rizzo NS, Sabate J, Jaceldo-Siegl K, Fraser GE. Vegetarian dietary patterns are associated with a lower risk of metabolic syndrome: the Adventist health study 2. Diabetes Care. 2011;34:1225-7. https://doi.org/10.2337/dc10-1221

15. Turner-McGrievy GM, Davidson CR, Wingard EE, Wilcox S, Frongillo EA. Comparative effectiveness of plant-based diets for weight loss: a randomized controlled trial of five different diets. Nutrition. 2015;31:350-8. https://doi.org/10.1016/j.nut.2014.09.002.

16. Orlich MJ, Singh PN, Sabaté J, Fan J, Sveen L, Bennett H, et al. Vegetarian dietary patterns and the risk of colorectal cancers. JAMA Intern Med. 2015; 175:767-76. https://doi.org/10.1001/jamainternmed.2015.59.

17. Fraser GE. Vegetarian diets: what do we know of their effects on common chronic diseases? Am J Clin Nutr. 2009;89:1607S-12S. https://doi.org/10. 3945/ajcn.2009.26736K

18. Yokoyama Y, Nishimura K, Barnard ND, Takegami M, Watanabe M, Sekikawa A, et al. Vegetarian diets and blood pressure: a meta-analysis. JAMA Intern Med. 2014;174:577-87. https://doi.org/10.1001/jamainternmed.2013.14547
19. Ford ES, Bergmann MM, Kröger J, Schienkiewitz A, Weikert C, Boeing $H$. Healthy living is the best revenge: findings from the European prospective investigation into Cancer and nutrition-Potsdam study. Arch Intern Med. 2009;169:1355-62. https://doi.org/10.1001/archinternmed.2009.237.

20. Phillips F. Vegetarian nutrition. Nutr Bull. 2005;30:132-67. https://doi.org/10. 1111/j.1467-3010.2005.00467.x.

21. Silva SCG, Pinho JP, Borges C, Santos CT, Santos A, Graça P. Guidelines for a healthy vegetarian diet. Lisbon: Direção-Geral da Saúde; 2015.

22. National Health and Medical Research Council and New Zealand Ministry of Health. Nutrient reference values for Australia and New Zealand including recommended dietary intakes. Canberra: NHMRC; 2006. Available from: http:// www.nhmrc.gov.au/guidelines/publications/n35-n36-n37 (Accessed 9 July 2018)

23. Richter $M$, Boeing $H$, Grünewald-Funk $D$, Heseker $H$, Kroke A, Leschik-Bonnet $E$, et al. Vegan diet. Position of the German nutrition society (DGE). Ernährungs Umschau. 2016:63(04):92-102. https://doi.org/10.4455/eu.2016.021.

24. Trapp D, Knez W, Sinclair W. Could a vegetarian diet reduce exerciseinduced oxidative stress? A review of the literature. J Sports Sci. 2010;28: 1261-8. https://doi.org/10.1080/02640414.2010.507676

25. Wirnitzer K, Seyfart T, Leitzmann C, Keller M, Wirnitzer G, Lechleitner C, et al. Prevalence in running events and running performance of endurance runners following a vegetarian or vegan diet compared to non-vegetarian endurance runners: the NURMI study. Springerplus. 2016;5:458. https://doi. org/10.1186/s40064-016-2126-4.

26. Lynch $\mathrm{H}$, Wharton C, Johnston C. Cardiorespiratory fitness and peak torque differences between vegetarian and omnivore endurance athletes: a crosssectional study. Nutrients. 2016;8:726. https://doi.org/10.3390/nu8110726 .

27. Nieman DC. Physical fitness and vegetarian diets: is there a relation? Am J Clin Nutr. 1999:70:570s-5s.

28. Hanne N, Dlin R, Rotstein A. Physical fitness, anthropometric and metabolic parameters in vegetarian athletes. J Sports Med Phys Fitness. 1986;26(2): 180-5.

29. Boldt P, Knechtle B, Nikolaidis P, Lechleitner C, Wirnitzer G, Leitzmann C, et al. Quality of life of female and male vegetarian and vegan endurance runners compared to omnivores - results from the NURMI study (step 2). J Int Soc Sports Nutr. 2018;15:33. https://doi.org/10.1186/s12970-018-0237-8

30. Wirnitzer K, Boldt P, Lechleitner C, Wirnitzer G, Leitzmann C, Rosemann T, et al. Health status of female and male vegetarian and vegan endurance runners compared to omnivores - results from the NURMI study (step 2). Nutrients. 2019;11:29. https://doi.org/10.3390/nu11010029.

31. Turner-McGrievy GM, Moore WJ, Barr-Anderson D. The interconnectedness of diet choice and distance running: results of the research understanding the NutritioN of endurance runners (RUNNER) study. Int J Sport Nutr Exerc Metab. 2015;26(3):205-11. https://doi.org/10.1123/ijsnem.2015-0085.

32. Wilson PB. Nutrition behaviors, perceptions, and beliefs of recent marathon finishers. Phys Sportsmed. 2016;44:242-51. https://doi.org/10.1080/00913847. 2016.1177477.

33. Deutsche Gesellschaft für Ernährung (DGE), Österreichische Gesellschaft für Ernährung (ÖGE), Schweizerische Gesellschaft für Ernährung (SGE) Referenzwerte für die Nährstoffzufuhr. 2. Auflage ed. Bonn: Neuer Umschau Buchverlag; 2018. 4. aktualisierte Ausgabe

34. Joint FAO/WHO/UNU Expert Consultation on Protein and Amino Acid Requirements in Human Nutrition (2002: Geneva, Switzerland), Food and Agriculture Organization of the United Nations, World Health Organization \& United Nations University. Protein and amino acid requirements in human nutrition: report of a joint FAO/WHO/UNU expert consultation. Geneva: World Health Organization; 2007. Available from: http://www.who. int/iris/handle/10665/43411

35. Kerksick CM, Wilborn CD, Roberts MD, Smith-Ryan A, Kleiner SM, Jäger R, et al. ISSN exercise \& sports nutrition review update: research \& recommendations. J Int Soc Sports Nutr. 2018;15:38. https:/doi.org/10.1186/s12970-018-0242-y.

36. Maughan RJ, Burke LM. In: Maughan RJ, Burke LM, editors. Practical nutritional recommendations for the athlete, vol. 69. Basel: KARGER; 2012. p. 131-49. https://doi.org/10.1159/000329292. Nestlé Nutr. Inst. Workshop Ser.

37. Thomas DT, Erdman KA, Burke LM. Position of the academy of nutrition and dietetics, dietitians of Canada, and the American College of Sports Medicine: nutrition and athletic performance. J Acad Nutr Diet. 2016;116: 501-28. https://doi.org/10.1016/j.jand.2015.12.006.

38. Loucks AB, Kiens B, Wright HH. Energy availability in athletes. J Sports Sci. 2011;29(Suppl 1):S7-15. https://doi.org/10.1080/02640414.2011.588958

39. Loucks $A B$. Low energy availability in the marathon and other endurance sports. Sports Med Auckl NZ. 2007;37:348-52. 
40. Costill D. Carbohydrates for exercise: dietary demands for optimal performance. Int J Sports Med. 1988;09:1-18. https://doi.org/10.1055/s2007-1024971.

41. Deriemaeker $P$, Alewaeters $K$, Hebbelinck M, Lefevre J, Philippaerts $R$, Clarys P. Nutritional status of Flemish vegetarians compared with nonvegetarians: a matched samples study. Nutrients. 2010;2:770-80. https:// doi.org/10.3390/nu2070770.

42. Schüpbach R, Wegmüller R, Berguerand C, Bui M, Herter-Aeberli I. Micronutrient status and intake in omnivores, vegetarians and vegans in Switzerland. Eur J Nutr. 2017;56:283-93. https://doi.org/10.1007/s00394015-1079-7.

43. Cade JE, Burley VJ, Greenwood DC, UK Women's Cohort Study Steering Group. The UK Women's cohort study: comparison of vegetarians, fisheaters and meat-eaters. Public Health Nutr. 2004;7:871-8.

44. Appleby PN, Thorogood M, Mann JI, Key TJ. The Oxford vegetarian study: an overview. Am J Clin Nutr. 1999;70:525S-31S

45. Tarnopolsky MA. Protein and physical performance. Curr Opin Clin Nutr Metab Care. 1999;2:533-7.

46. Tarnopolsky MA, MacDougall JD, Atkinson SA. Influence of protein intake and training status on nitrogen balance and lean body mass. J Appl Physiol. 1988:64:187-93. https://doi.org/10.1152/jappl.1988.64.1.187.

47. Boisseau N, Creff CL, Loyens M, Poortmans JR. Protein intake and nitrogen balance in male non-active adolescents and soccer players. Eur J Appl Physiol. 2002;88:288-93. https://doi.org/10.1007/s00421-002-0726-X.

48. Davey GK, Spencer EA, Appleby PN, Allen NE, Knox KH, Key TJ. EPIC-Oxford: lifestyle characteristics and nutrient intakes in a cohort of 33883 meateaters and 31546 non meat-eaters in the UK. Public Health Nutr. 2003;6. https://doi.org/10.1079/PHN2002430.

49. Sundgot-Borgen J, Meyer NL, Lohman TG, Ackland TR, Maughan RJ, Stewart $A D$, et al. How to minimise the health risks to athletes who compete in weight-sensitive sports review and position statement on behalf of the ad hoc research working group on body composition, health and performance, under the auspices of the IOC medical commission. $\mathrm{Br} J$ Sports Med. 2013;47:1012-22. https://doi.org/10.1136/bjsports-2013-092966.

50. International Society for the Study of Fatty Acids and Lipids. Recommendations for intake of polyunsaturated fatty acids in healthy adults, 2004

51. Welch AA, Shakya-Shrestha S, Lentjes MA, Wareham NJ, Khaw K-T. Dietary intake and status of $n-3$ polyunsaturated fatty acids in a population of fisheating and non-fish-eating meat-eaters, vegetarians, and vegans and the precursor-product ratio of a-linolenic acid to long-chain $n-3$ polyunsaturated fatty acids: results from the EPIC-Norfolk cohort. Am J Clin Nutr. 2010:92:1040-51. https://doi.org/10.3945/ajen.2010.29457.

52. Max-Rubner Institut. Nationale Verzehrsstudie II. Ergebnisbericht Teil II. In: Die bundesweite Befragung zur Ernährung von Jugendlichen und Erwachsenen. Karlsruhe: Max-Rubner Institut; 2008

53. Mensink G, Ströbel A. Einnahme von Nahrungsergänzungspräparaten und Ernährungsverhalten. Gesundheitswesen. 1999;61(\$2):132-7.

54. Faude O, Fuhrmann M, Herrmann M, Kindermann W, Urhausen A. Ernährungsanalysen und Vitaminstatus bei deutschen Spitzenathleten. Leistungssport. 2005;35(4):4-9.

55. Baranauskas M, Stukas R, Tubelis L, Žagminas K, Šurkienė G, Švedas E, et al. Nutritional habits among high-performance endurance athletes. Med Kaunas Lith. 2015;51:351-62. https://doi.org/10.1016/j.medici.2015.11.004.

56. European Food Safety Authority. Tolerable upper intake levels for vitamins and minerals. Parma: European Food Safety Authority; 2006.

57. Institute of Medicine (US) Standing Committee on the Scientific Evaluation of Dietary Reference Intakes and its Panel on Folate, Other B Vitamins, and Choline. Dietary Reference Intakes for Thiamin, Riboflavin, Niacin, Vitamin B6, Folate, Vitamin B12, Pantothenic Acid, Biotin, and Choline. Washington (DC): National Academies Press (US); 1998.

58. Kristensen NB, Madsen ML, Hansen TH, Allin KH, Hoppe C, Fagt S, et al. Intake of macro- and micronutrients in Danish vegans. Nutr J. 2015;14 https://doi.org/10.1186/s12937-015-0103-3.

59. Elorinne A-L, Alfthan G, Erlund I, Kivimäki H, Paju A, Salminen I, et al. Food and nutrient intake and nutritional status of Finnish vegans and nonvegetarians. PLOS one. 2016;11:e0148235. https://doi.org/10.1371/journal. pone.0148235

60. Eisinger $M$, Plath $M$, Jung $K$, Leitzmann C. Nutrient intake of endurance runners with ovo-lacto-vegetarian diet and regular western diet. Z Ernahrungswiss. 1994;33:217-29.
61. Waldmann A, Koschizke JW, Leitzmann C, Hahn A. German vegan study: diet, life-style factors, and cardiovascular risk profile. Ann Nutr Metab. 2005; 49:366-72. https://doi.org/10.1159/000088888.

62. Pilz S, Zittermann A, Trummer C, Schwetz V, Lerchbaum E, Keppel M, et al. Vitamin $D$ testing and treatment: a narrative review of current evidence. Endocr Connect. 2019. https://doi.org/10.1530/EC-18-0432.

63. Lun V, Erdman KA, Reimer RA. Evaluation of nutritional intake in Canadian high-performance athletes. Clin J Sport Med. 2009;19:405. https://doi.org/10. 1097/JSM.0b013e3181b5413b.

64. Hinton PS. Iron and the endurance athlete. Appl Physiol Nutr Metab. 2014; 39:1012-8. https://doi.org/10.1139/apnm-2014-0147.

65. Gropper SS, Blessing D, Dunham K, Barksdale JM. Iron status of female collegiate athletes involved in different sports. Biol Trace Elem Res. 2006; 109:1-14. https://doi.org/10.1385/BTER:109:1:001.

66. West A-R, Oates P-S. Mechanisms of heme iron absorption: current questions and controversies. World J Gastroenterol. 2008;14:4101-10.

67. Anderson GJ, Frazer DM, McKie AT, Vulpe CD, Smith A. Mechanisms of haem and non-haem iron absorption: lessons from inherited disorders of iron metabolism. Biometals. 2005;18:339-48. https://doi.org/10.1007/ s10534-005-3708-8.

68. Tenforde AS, Sayres LC, Sainani KL, Fredericson M. Evaluating the relationship of calcium and vitamin $D$ in the prevention of stress fracture injuries in the young athlete: a review of the literature. PM R. 2010;2:945-9. https://doi.org/10.1016/j.pmrj.2010.05.006.

69. Chatard JC, Mujika I, Guy C, Lacour JR. Anaemia and iron deficiency in athletes. Practical recommendations for treatment. Sports Med. 1999:27: 229-40. https://doi.org/10.2165/00007256-199927040-00003.

70. Gröber U. Mikronährstoffe im Leistungssport. Deutsche Apotheker Zeitung, vol. 23; 2012. p. 86-92.

71. Brouns F. Heat--sweat--dehydration--rehydration: a praxis oriented approach J Sports Sci. 1991;9:143-52. https://doi.org/10.1080/02640419108729871

72. Mao IF, Chen ML, Ko YC. Electrolyte loss in sweat and iodine deficiency in a hot environment. Arch Environ Health. 2001;56:271-7. https://doi.org/10. 1080/00039890109604453

\section{Publisher's Note}

Springer Nature remains neutral with regard to jurisdictional claims in published maps and institutional affiliations.

Ready to submit your research? Choose BMC and benefit from:

- fast, convenient online submission

- thorough peer review by experienced researchers in your field

- rapid publication on acceptance

- support for research data, including large and complex data types

- gold Open Access which fosters wider collaboration and increased citations

- maximum visibility for your research: over $100 \mathrm{M}$ website views per year

At $\mathrm{BMC}$, research is always in progress.

Learn more biomedcentral.com/submissions 